\title{
mTOR Signal Transduction Pathways Contribute to TN-C FNIII A1 Overexpression by Mechanical Stress in Osteosarcoma Cells
}

\author{
Lianhe Zheng, Dianzhong Zhang, Yunfei Zhang, Yanhua Wen, and Yucai Wang*
}

\begin{abstract}
Osteosarcoma is the most common primary malignant bone tumor with a very poor prognosis. Treating osteosarcoma remains a challenge due to its high transitivity. Tenascin- $C$, with large molecular weight variants including different combinations of its alternative spliced FNIII repeats, is specifically over expressed in tumor tissues. This study examined the expression of Tenascin-C FNIIIA1 in osteosarcoma tissues, and estimated the effect of mechanical stimulation on A1 expression in MG-63 cells. Through immunohistochemical analysis, we found that the A1 protein was expressed at a higher level in osteosarcoma tissues than in adjacent normal tissues. By cell migration assay, we observed that there was a significant correlation between A1 expression and MG-63 cell migra-tion. The relation is that Tenascin-C FNIIIA1 can promote MG-63 cell migration. According to our further study into the effect of mechanical stimulation on A1 expression in MG-63 cells, the mRNA and protein levels of A1 were significantly up-regulated under mechanical stress with the mTOR molecule proving indispensable. Meanwhile, 4E-BP1 and S6K1 (downstream molecule of mTOR) are necessary for A1 normal expression in MG-63 cells whether or not mechanical stress has been encountered. We found that Tenascin-C FNIIIA1 is over-expressed in osteosar-coma tissues and can promote MG-63 cell migration. Furthermore, mechanical stress can facilitate MG-63 cell migration though facilitating $A 1$ overexpression with the necessary molecules (mTOR, 4E-BP1 and S6K1). In con-clusion, high expression of A1 may promote the meta-stasis of osteosarcoma by facilitating MG-63 cell migration. Tenascin-C FNIIIA1 could be used as an indicator in metastatic osteosarcoma patients.
\end{abstract}

\footnotetext{
Department of Orthopaedic Surgery, Tangdu Hospital, Fourth Military Medical University, Xi'an, 710032, Shaanxi Province, China

*Correspondence: yucwangxian @gmail.com
}

Received 5 September, 2013; revised 13 January, 2014; accepted 14 January, 2014; published online 19 February, 2014

Keywords: mechanical stimulation, MG-63 cells, osteosarcoma, tenascin-C, TN-C FNIIIA1

\section{INTRODUCTION}

Osteosarcoma (OS) is the most common primary malignant bone tumor, arising from the malignant transformation of mesenchymal cells, which differentiate towards the formation of osteoid and bone (Lv et al., 2013; Osborne and Khanna, 2012). Conventionally, according to histological features, OS is classified into chondroblastic, osteoblastic and fibroblastic OS (Bielack et al., 2009). The incidence is higher in adolescents, where it accounts for $>10 \%$ of all solid cancers and $20-35 \%$ of all malignant primary bone tumors (Bielack et al., 2009; Rhee et al., 2013). OS usually arises in the metaphysis of a long extremity bone, most commonly around the knee (Bielack et al., 2009); it is highly aggressive and mostly metastasizes to the bones and lungs (Gill et al., 2012). However, despite the use of aggressive chemotherapeutic treatment strategies, the survival rate of OS patients has still not been improved. Also, the prognosis is very poor, especially in patients with clinically detectable metastasis at diagnosis (Wachtel and Schäfer, 2010). Osteosarcoma is usually associated with aggressive behavior and a high degree of local recurrence and metastasis (Rhee et al., 2013). The most significant negative prognostic factor in the treatment of tumors is the development of metastatic disease. As a result, treating metastatic osteosarcoma remains a challenge for oncologists (Osborne and Khanna, 2012). Thus, a novel strategy to efficiently inhibit the metastasis of osteosarcoma is highly indispensable.

The extracellular matrix (ECM) contains a series of important cues for tissue homeostasis and morphogenesis (Alves et al., 2011). Interactions between cells and the ECM also regulate the ability of a cell to proliferate, migrate, and differentiate (Lelievre et al., 2000). Most ECM glycoproteins promote cell adhesion and cause cytoskeletal reorganization, leading to signals that direct differentiation and promote cell survival (MurphyUllrich, 2001). Another class of ECM proteins exists, termed "matricellular" proteins, which function as adaptors and modulators of cell-matrix interactions (Sage and Bornstein, 1991). These structurally diverse proteins include thrombospondins (TSPs) and tenascins, both of which exhibit highly regulated expression during development and cellular injury (MurphyUllich, 2001).

Tenascins are a family of four multimeric extracellular matrix (ECM) glycoproteins, including tenascin-C, X, R and W (Tucker et al., 2006). Studies have shown that the matricellular protein 
tenascin- $\mathrm{C}(\mathrm{TN}-\mathrm{C})$ is overexpressed in remodeling tissues and tumors (Alves et al., 2011). For instance, tenascin-C is highly expressed in glioblastoma multiforme (GBM) (Leins et al., 2003) and giant cell tumors of bones (Pazzaglia et al., 2010). In addition, TN-C can also facilitate cell migration and proliferation (Murphy-Ullrich, 2001). In the last decade, it has been demonstrated that tenascin-C plays a vital role in cardiac and arterial injury, tumor angiogenesis and metastasis (Midwood et al., 2011). A recent study also showed that tenascin-C knock-down in the human LN229 GBM cell line had no significant effect on cell proliferation in vitro but abolished cell migration by promoting focal adhesion disassembly signals (Hirata et al., 2009; Midwood et al., 2011).

Tenascin- $C$ is composed of four distinct domains: an assembly domain, a series of epidermal growth factor-like repeats (EGF-L), a series of fibronectin type III-like repeats (FNIII), and a C-terminal globular domain homolog to fibrinogen $\beta$ - and $\gamma$ chains (Midwood and Orend, 2009; Midwood et al., 2011). The alternatively spliced FNIII repeats, which includes A1-A4, B, $A D 2, A D 1, C$ and $D$ subdomains in turn, were shown to promote the migration of cardiac fibroblasts, suggesting that this domain is responsible for the observed tenascin- $C$ effect (Midwood et al., 2011; Tamaoki et al., 2005). Among these domains, FNIIIA1 participate in inhibiting lymphocyte activation, tumor infiltrating lymphocytes and proliferation, as well as blocking the secretion of cytokines (Midwood and Orend, 2009).

There are many factors which have been shown to facilitate tenascin- $C$ expression, including growth factors, oxidative stress, inflammatory cytokines and mechanical stress (Tucker and Chiquet-Ehrismann, 2009). Among these factors, research has shown that mechanical stimulation is necessary for normal skeletal development. Reduced loading due to long-term bed rest, cast immobilization, or microgravity conditions induces significant bone loss and mineral changes, which only begin to be recovered following the reintroduction of normal activity (Ehrlich and Lanyon, 2002). However, it remains unknown whether mechanical stimulation can promote MG-63 cells migration through facilitating the overexpression of FNIIIA1 of tenascin C. In this study, we chose osteosarcoma tissue and the MG-63 cell line to explore the effect of mechanical stress on the migration of MG-63 cells. Our results suggest that FNIIIA1 exhibits high expression in osteosarcoma tissue and facilitates the migration of MG-63 cells. Furthermore, with the action of mechanical stress, overexpression of FNIIIA1 was achieved by mTOR, 4EBP1 and S6K1.

\section{MATERIALS AND METHODS}

Dulbecco's modified Eagle's medium (DMEM), fetal bovine serum (FBS), TRIzol, silicone membrane and Lipofectamine ${ }^{T M}$ 2000 were purchased from Invitrogen Life Technologies (USA). Anti- $\beta$-actin antibody was purchased from Santa Cruz Biotechnology (USA). Bovine serum albumin (BSA), PVDF membranes, protease inhibitor cocktail, RNase inhibitor, Tween20, RIPA buffer, ascorbic acid, Harris hematoxylin solution, gentamicin, penicillin, mitomycin $C$ and Goat serum were purchased from Sigma-Aldrich (USA). GeneSilencer siRNA transfection reagent was purchased from Gene Therapy System (USA). DNase, RT-PCR kit, and Qiagen RNeasy kit were purchased from Qiagen (Hilden, Germany). LumiGLo reagent and enhanced chemiluminescence (ECL) were purchased from Thermo Pierce (USA). The SYBR ${ }^{\circledR}$ Green PCR Master Mix was purchased from Applied Biosystems (USA). The Diaminobenzidine (DAB) stain kit was purchased from Vector Laboratories,
Inc. (USA). Rapamycin (mTOR inhibitor) was purchased from Cell Signaling Technology Inc. (USA). Trypsin-EDTA solution was purchased from Life Technologies (USA). Pyrobest DNA polymerase and avian myeloblastosis virus (AMV) reverse transcriptase were purchased from Takara Biotechnology (Dalian, China). HRP-conjugated sheep anti-mouse IgG antibody was purchased from Cell Signaling Technology (USA). Enhanced Fluorescent Protein Vector (pEGFP-N1) was purchased from Novagen (USA). Anti-annexin II antibody was purchased from BD Biosciences (USA).

\section{Immunohistochemical analysis}

This study was approved by the Ethics Committee of the Fourth Military Medical University in accordance with the Declaration of Helsinki. The 54 osteosarcoma tissue samples and 12 adjacent normal tissue samples were collected from osteosarcoma patients by Tangdu Hospital of the Fourth Military Medical University between May 2008 and February 2012. All of the patients had not received any radiation treatment or chemotherapy before surgery. These tissues were fixed in 30\% formalin, dehydrated in ethanol, transparentized in ethanol and dimethylbenzene, and embedded in paraffin wax (Wu et al., 2013). All specimens were sliced continuously into $3 \mu \mathrm{m}$ sections. Paraffinembedded sections were washed in PBS and blocked with $5 \%$ normal goat serum in incubation buffer $(0.2 \%$ Tween20 and $0.1 \%$ BSA in PBS). Incubation with mouse anti-human Tenascin-C FNIII domain A1 antibodies, which were prepared according to the method described previously (Wang, 2010), was performed in a humidified chamber overnight at $4^{\circ} \mathrm{C}$. Sections were subsequently washed in washing buffer $(0.5 \%$ Tween20 in PBS) and incubated with HRP-conjugated sheep anti-mouse IgG antibodies. A diaminobenzidine (DAB) stain kit was used to detect a positive reaction by producing a brown color.

\section{Culture of MG-63 cells}

MG-63 cells purchased from the American type culture collection (ACTT) were cultured in DMEM culture solution supplemented with $10 \%$ FBS, $50 \mu \mathrm{g} / \mathrm{ml}$ ascorbic acid, $100 \mu \mathrm{g} / \mathrm{ml}$ gentamycin and $100 \mathrm{unit} / \mathrm{ml}$ penicillin at $37^{\circ} \mathrm{C}$ in a humidified atmosphere containing $5 \% \mathrm{CO}_{2}$. During this process, culture solution was changed every 3 days. Adherent cells were digested by $0.25 \%$ Trypsin-EDTA solution when the confluence reaches 90\% (Andrianarivo et al., 1992; Tamaoki et al., 2005).

\section{Construction and transfection of tenascin-C FNIII A1 expression vector}

The CDNA of FNIIIA1 was sub-cloned with pyrobest DNA polymerase and inserted into a pEGFP-N1 vector. These vectors were transfected into MG-63 cells using Lipofectamine ${ }^{T M} 2000$ (Saito et al., 2007). In addition, MG-63 cells were transferred by pEGFP-N1 using Lipofectamine ${ }^{\mathrm{TM}} 2000$ as the control group. All of the cells were used for experiments at $72 \mathrm{~h}$ after transfection.

\section{Silencing of tenascin-C FNIIIA1 expression with small} interference RNA (siRNA)

To design target-specific siRNA, an siRNA that was specific for the FNIIIA1 and a scramble control were designed according to the cDNA sequences using the internet tool of siRNA wizard (InvivoGen). The sequences were: 5'-GAA CCA GGA CAG GAG UAC AAU-3' (21-nt at position 37-57 relative to the start codon) for the FNIIIA1 and 5'-GGA AAC GAC GGA AAC CAG UUA-3' (21-nt) for the scramble control. The selected siRNA sequences were also submitted to BLAST searches. The sense and antisense strands with two base overhangs were 
chemically synthesized and annealed (GeneChem, China). MG-63 cells $\left(6 \times 10^{5}\right.$ cells $\left./ \mathrm{ml}\right)$ were transfected with $2 \mu \mathrm{g} / \mathrm{ml}$ FNIIIA1-siRNA or Scramble siRNA using the GeneSilencer siRNA transfection reagent (GeneTherapy System), according to manufacturer's instructions. Twenty-four hours post-transfection, the cells were washed with PBS (Feng et al., 2010).

\section{Cell migration assay}

Migration studies of MG-63 cells (tenascin-C FNIIIA1 overexpression, tenascin-C FNIIIA1 low expression or normal) were performed as previously described (Li et al., 2007). Cell cultures $\left(2.5 \times 10^{6}\right.$ cells/well) were seeded into 6 -well tissue culture plates and were allowed to grow to post-confluence. Two days later, cell proliferation was suppressed by incubation with 10 $\mathrm{mg} / \mathrm{L}$ mitomycin $\mathrm{C}$ for $2 \mathrm{~h}$ before the experiment. Then, 2 denuded areas $(10 \times 5 \mathrm{~mm})$ were made in each well using a custom-designed cell scraper. The MG-63 cells were subsequently allowed to migrate for $12 \mathrm{~h}$. After incubation for the indicated time, the cells were fixed with cold methanol at $4^{\circ} \mathrm{C}$ for $30 \mathrm{~min}$ and were stained with Harris hematoxylin solution at room temperature for $40 \mathrm{~min}$. Migration was quantified by counting the average number of cells that migrated into the denuded area in 5 microscope fields covering the border of the entire denuded area. Each assay was repeated at least 3 times (Si et al., 2013). In addition, tenascin-C FNIIIA1 overexpression MG-63 cells treated with or without anti- annexin II antibody were subject to cell migration assay as described above.

\section{Coating of silicone membranes with collagen and mechanical stress assay}

Three Dimention collagen cell culture system was established as previously described (Wang et al., 2006). In brief, a sterile solution of collagen was neutralized with $0.1 \%$ acetic acid and diluted to a final concentration of $150 \mu \mathrm{g} / \mathrm{ml}$ with $0.01 \mathrm{M}$ ice-cold PBS. A $100-\mu \mathrm{l}$ drop of sterile collagen solution was carefully placed onto the silicone membrane $(0.05 \mathrm{U}$, gloss/gloss) at the bottom of a six-well plate as previously described (Jiang et al., 2008). Collagen was allowed to adsorb to the silicone membrane at room temperature for $4 \mathrm{~h}$. Before seeding cells, redundant liquid was removed, and collagen-coated wells were kept under a UV lamp (254 nm, $20 \mathrm{~W}, 15-\mathrm{cm}$ distance) for 30 min until silicone membrane dried.

Specially designed six-well dishes prepared as above covered by MG-63 were mounted onto a vacuum extraction device (Developed by Xi'an Jiaotong University, China). Cells were directly subjected to cyclic strain $(10 \%, 0.2 \mathrm{~Hz})$ for indicated time $(0 \mathrm{~h}, 1 \mathrm{~h}, 4 \mathrm{~h}, 8 \mathrm{~h}$, and $12 \mathrm{~h})$. For different experiments, cells were divided into different groups.

\section{Cell interference experiment}

In this experiment, we chose $0.2 \mathrm{~Hz}$ as the stimulating frequency according to our pre-experiment investigations. The grown MG-63 cells were digested by $0.25 \%$ Trypsin-EDTA solution and resuspended in DMEM culture solution. Then, $6 \times 10^{5} /$ well MG-63 cells $(1 \mathrm{ml})$ were plated on collagen-coated silicone membrane in 6-well plates per-treated above. The MG-63 cell cultures were divided into 4 groups: control group (cells without any addition) and experimental groups including treatment with mTOR inhibitor, 4E-BP1 siRNA or S6K1 siRNA, respectively. The latter groups were treated differently before receiving mechanical stimulation. After MG-63 cells showed confluence, cells were cultured with serum-free DEME culture solution and $1 \%$ BSA for $24 \mathrm{~h}$ in order to implement cell synchronization. Then, the plates covered by MG-63 cells were mounted onto the vacuum extraction device. MG-63 cells were directly subjected to mechanical stress $(0.2 \mathrm{~Hz})$ for indicated time $(0,1,4,8$, $12 \mathrm{~h}$ ) and then were lysed for qRT-PCR and Western blotting to detect the expressions of TN-C FNIIIA1. MG-63 cells at resting were lysed at the same time for detecting TN-C FNIIIA1. The siRNA sequences were synthesized by GeneChem (Shanghai, China) and the sequences identified were as follows: S6K1 siRNA (target sequence: 5'-AAC AGU GGA GAA CUA UUU$3^{\prime}$ ) and a negative control siRNA (nonspecific target sequence: 5'-AAU AGC GAC UAA ACA CAU CAA-3'); 4E-BP1 siRNA (target sequence: 5'-UCU AUG ACC GGA AAU UCC UGA UGG $A-3^{\prime}$ ) and a negative control siRNA (nonspecific target sequence: 5'-UCU CCA GAA GGC UUA AGU CUU AGG A-3').

\section{qRT-PCR}

Total RNA was extracted with TRIzol and purified with the Qiagen RNeasy kit according to the manufacturer's instructions, followed by treatment with DNase. The levels of gene mRNA transcripts were analyzed by one-step quantitative reverse transcriptase-polymerase chain reaction (RT-PCR) using the specific primers and the SYBR ${ }^{\circledR}$ Green PCR Master Mix and RT-PCR kit according to the manufacturer's instructions, on an ABI Prism 7700 Sequence Detection System. Primers specific for human FNIIIA1, A2, A3, A4 of TN-C and $\beta$-actin were synthesized by the Shanghai Sangon Biological Engineering and Technology Service (China). The sequences of primers were as follows: 5'-GCT TCC AAG AAA CAC TTC CTA T-3' (senseprimer) and 5'-CCC GAA GGC TGC CAG GCA CGG TGA G$3^{\prime}$ (anti-sense primer) for Tenascin-C FNIIIA1, 5'-AAC CTC ACC GTG CCT GGC AGC CTT C-3' (sense-primer) and 5'CTC CTG GGA CGG TGA GGT TCT GGG C-3' (anti-sense primer) for Tenascin-C FNIIIA2, 5'-CCA GAA CCT CAC CGT CCC AG-3' (sense-primer) and 5'-CTG CCA GGA ACC GTG AGA TTG TGA G-3' (anti-sense primer) for Tenascin-C FNIIIA3, 5'-CAC AAT CTC ACG GTT CCT GGC AGC C-3' (senseprimer) and 5'-CTG AGG CTG CCA GGC AAC GTG AGG T-3' (anti-sense primer) for Tenascin-C FNIIIA4. Briefly, $20 \mu \mathrm{l}$ reactions containing $50 \mathrm{ng}$ of total RNA, $10 \mu \mathrm{l} 2 \times$ SYBR Green PCR Master Mix, 6.25 U AMV reverse transcriptase, $10 \cup$ RNase inhibitor and $0.1 \mathrm{mM}$ primers were subjected to one cycle of $95^{\circ} \mathrm{C}$ for $10 \mathrm{~min}$ and then 40 cycles of $95^{\circ} \mathrm{C}$ for $15 \mathrm{~s}, 56^{\circ} \mathrm{C}$ for $30 \mathrm{~s}$ and $72^{\circ} \mathrm{C}$ for $45 \mathrm{~s}$. For relative quantification, the levels of individual gene mRNA transcripts were firstly normalized to control $\beta$-actin (sense: 5'-CTC CAT CCT GGC CTC GCT GT-3', anti-sense: 5'-GCT GTC ACC TTC ACC GTT CC-3'). Subsequently, the differential expression of these genes was analyzed by the $\Delta \mathrm{Ct}$ method and expressed as fold-changes (Feng et al., 2010; Skelly et al., 2013).

\section{Western blotting}

MG-63 cells (treatment groups or control groups) were lysed with RIPA buffer. The protein concentration was determined using the BCA assay. The protein lysates ( $40 \mu \mathrm{g} /$ lane) were separated by SDS-PAGE on $10 \%$ polyacrylamide gels and transferred onto PVDF membranes. After blocking with $0.5 \%$ skimmed milk powder in $1 \times$ PBS-Tween20, the target proteins were probed with 1:1000 mouse anti-human Tenascin-C FNIII domain $\mathrm{A} 1$ or anti- $\beta$-actin antibody overnight at $4^{\circ} \mathrm{C}$. After washing thrice (10 min/time) with $1 \times$ PBS-Tween20, the membranes were incubated with HRP-conjugated sheep anti-mouse IgG antibodies (1:4000 dilution by PBS) at room temperature for $1 \mathrm{~h}$ and washed again. The reactive bands were detected by enhanced chemiluminescence (ECL) according to the manufacturer's protocol and the relative levels of each protein to $\beta$ - 

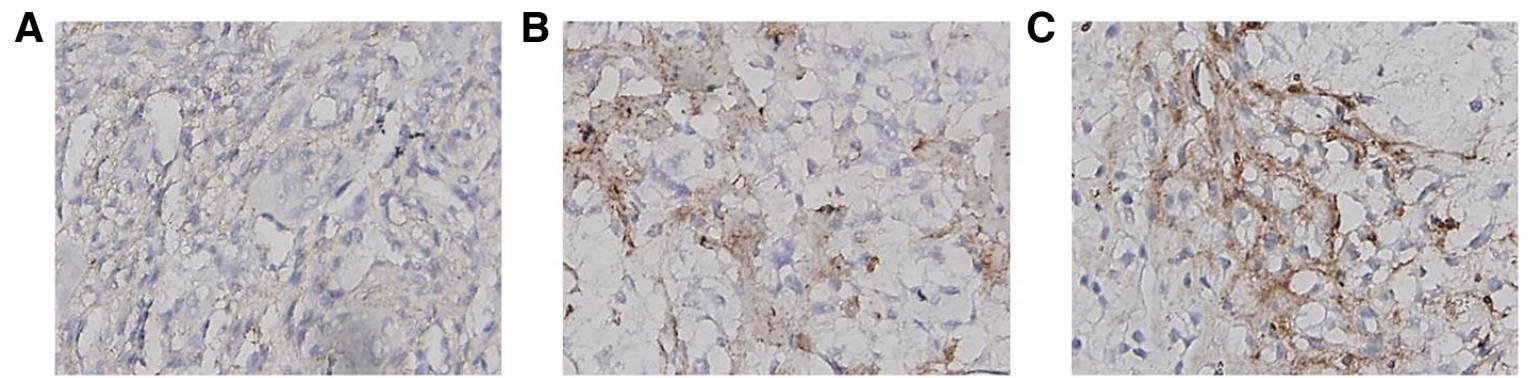

Fig. 1. Overexpression of TN-C FNIIIA1 in OS tissues. TN-C FNIIIA1 in the extracellular matrix (ECM) of normal tissues and OS tissues was detected by Immunohistochemistry staining. (A) normal tissue; (B) OS tissue; (C) OS tissue. Brown color indicates positive expression of A1. Magnification, $\times 40$.

actin were analyzed (Shimoyama et al., 2010).

\section{RESULTS}

Overexpression of TN-C FNIII A1 in OS tissues

Previous studies have shown that matricellular protein tenascin-C (TN-C) is overexpressed in various tumor tissues (Alves et al., 2011). However, expression of the FNIII A1 domain of TN-C in tumors is still barely understood, especially in osteosarcoma tissues. In this study, we compared the FNIIIA1 expression between osteosarcoma and adjacent normal tissues by immunohistochemistry. As shown in Fig. 1, the up-regulation of $\mathrm{A} 1$ in OS tissues (Figs. $1 \mathrm{~B}$ and $1 \mathrm{C}$ ) was found to be significant compared with normal tissues (Fig. 1A). This result suggests that TN-C FNIIIA1 is over expressed in OS tissues.

\section{TN-C FNIIIA1 facilitates MG-63 cell migration}

The above result indicates that TN-C FNIIIA1 is over expressed in OS tissues. In order to explore the function of A1 for MG-63 cells, we constructed A1-overexpression and A1-knockdown MG-63 cells, and these two cell models were verified by qRTPCR and Western blotting. As shown in Fig. 2A, the mRNA level of $A 1$ was significantly up-regulated in $A 1$ overexpression MG-63 cells and down-regulated in A1 knockdown MG-63 cells compared with the control group $\left({ }^{*} P<0.05\right)$. Also, the same results at the protein level for $A 1$ by western blotting are shown in Fig. 2B.

Then through the cell migration assay, we analyzed the effect of $A 1$ on MG-63 cell migration. Cells migrating into the denuded area were counted at the indicated time. As shown in Fig. $2 \mathrm{C}$, the number of MG-63 cells entering the scratched area increased with time. The cell intensity of the area around scratches decreased and cell spacing became larger with a loss of contact with the neighboring cells due to cell migration over time. In addition, the number of MG-63 cells migrating into the denuded area increased with the order of $A 1$ knockdown MG63 cells, MG-63 cells at resting (control) and A1 overexpression MG-63 cells (Fig. 2C). These results suggest that TN-C FNIIIA1 can induce MG-63 cell migration.

Previous studies by Chung CY have reported that cell surface annexin II is a high affinity receptor for the alternatively spliced segment of tenascin- $\mathrm{C}$, and may mediate cellular responses to soluble TN-C in the extracellular matrix. They found that antibodies to annexin II prominently stained the external surface of live endothelial cells and blocked the binding of TN-C FNIIIA-D to the cells (Chung and Erickson, 1994). Based on this discovery, we designed another experiment for cell migra- tion assay as described above in order to explore whether can TN-C FNIIIA1 affect MG-63 cell migration though its cell surface annexin II. Figure 2D shows that the migration of TN-C FNIIIA1 overexpression MG-63 cells treated with anti-annexin II antibody is significantly inhibited compared with control group. This result suggest that annexin II is necessary for A1-overexpression mediated MG- 63 cells migration, which may be the receptor for the alternatively spliced segment of TN-C FNIIIA1.

\section{Overexpression of TN-C FNIIIA1 in MG-63 cells}

Studies have shown that TN-C can facilitate cell migration, with the alternatively spliced FNIII repeats of TN-C being shown to be responsible for this effect (Midwood et al., 2011; MurphyUllrich, 2001; Tamaoki et al., 2005). In addition, mechanical stress has been shown to facilitate TN-C expression (Tucker and Chiquet-Ehrismann, 2009). In this study, we found that TNC FNIIIA1 was over-expressed in OS tissues. However, it is still unknown whether mechanical stress can influence the expression of $A 1$. In order to clarify this question, we detected variations of A1 expression in MG-63 cells which were exposed to mechanical stress in vitro. In this section, we mainly explored the expression of $A 1$ in MG-63 cells under mechanical stress. Analysis of $\mathrm{A} 1$ expression by qRT-PCR was as described above. As shown in Fig. 3 , the mRNA level of $A 1$ was significantly up-regulated $(1 \mathrm{~h}, 4 \mathrm{~h}, 8 \mathrm{~h}$ and $12 \mathrm{~h})$ compared with $0 \mathrm{~h}$ ( $\left.{ }^{\star} P<0.05\right)$, especially at $12 \mathrm{~h}$. However, there is no significant variation of $A 2, A 3$ and $A 4$.

\section{Inhibition of mTOR decreases TN-C FNIIIA1 expression in MG-63 cells}

mTOR is a central controller of cell growth and survival in response to growth factors, cytokines, hormones and nutrients (Wullschleger et al., 2006). Studies have shown that genetic aberrations of the PI3K-mTOR pathway are among the most common events in human malignancies, resulting in hyperactivation of mTOR signaling and causing these cancer cells highly addictive to mTOR pathway (Yuan and Cantley, 2008). mTOR is a PI3K-related kinase that forms two distinct protein complexes called mTORC1and mTORC2 (Oh and Jacinto, 2011). In response to upstream stimuli, mTORC1 phosphorylates S6K1 and 4E-BP1 to stimulate protein synthesis (Ma and Blenis, 2009; Zhang and Zheng, 2012).

In order to explore the effect of mTOR on A1 expression, we detected mRNA and protein levels of A1 in MG-63 cells with pretreatment with mTOR inhibitors, mechanical stress, or both, respectively. As shown in Fig. 4A, the mRNA of $\mathrm{A} 1$ was signifycantly up-regulated in the group which only received mechani- 
mTOR Contribute to TN-C FNIII A1 Overexpression

Lianhe Zheng et al.

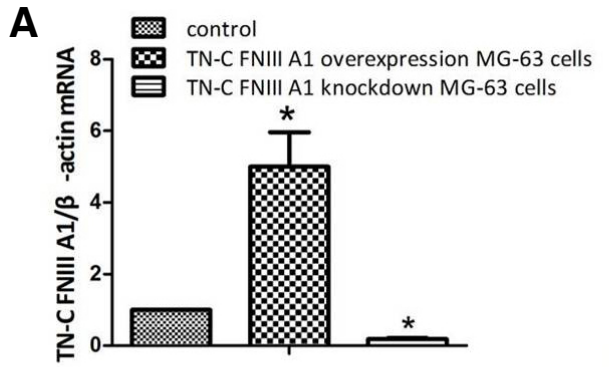

C

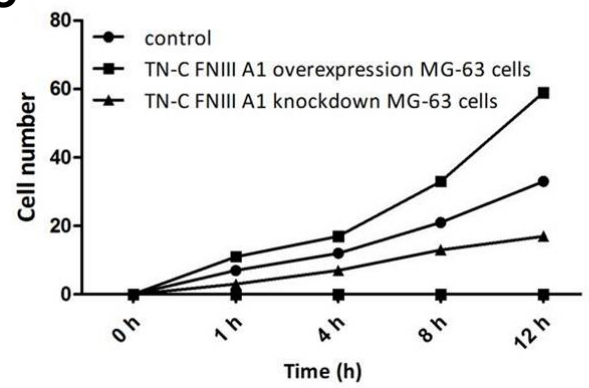

B

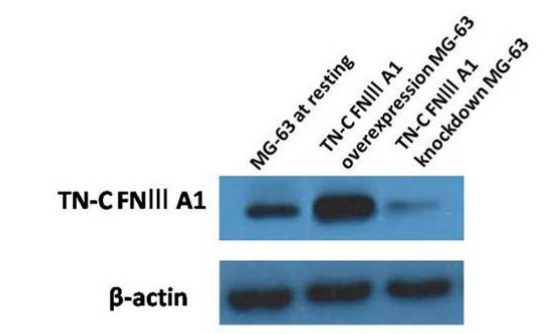

D

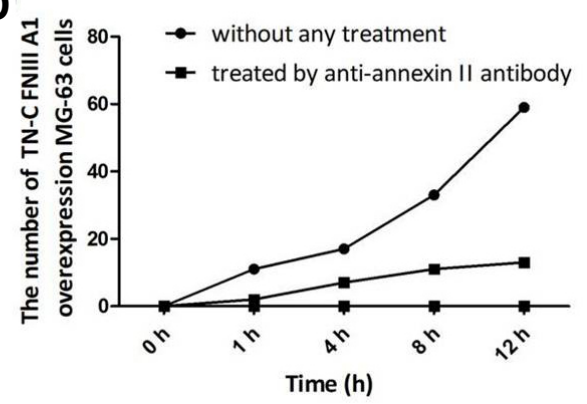

Fig. 2. TN-C FNIIIA1 facilitates MG-63 cell migration. The pEGFPN1-TN-C FNIIIA1 recombinants was transfected into MG-63 cells by liposome transfection. And $\mathrm{A} 1$ knockdown MG-63 cells were constructed by RNA interference. A1 expressions in these cells were verified by qRT-PCR and Western blotting, and cellular mig-ration abilities were detected by cell migration assay. Data were normalized based on the $\beta$ actin levels. (A) The expression of A1 mRNA by qRT-PCR. ${ }^{*} \mathrm{P}<$ 0.05 by Student's test when compared with control group; (B) The protein level of $A 1$ by Western blotting. Besides, the effect of A1 on MG-63 cell migration was detected by cell migration assay. (C) The number of MG-63 cells migrating into the denuded area. (D) The number of TN-C FNIIIA1 overexpression MG-63 cells migrating into the denuded area. cal stress and was down-regulated in the group that was only pretreated by mTOR inhibitor. Both of these groups were compared with the control group ( $\left.{ }^{*} P<0.05\right)$, and there was a significant difference also between the two groups ( ${ }^{*} P<0.05$ ). The same results at the protein level by Western blotting are shown in Fig. 4B. These results suggest that mechanical stimulation can promote the expression of $A 1$ in MG-63 cells through mTOR.

\section{Silencing of 4E-BP1 and S6K1 decreases the expression of} TN-C FNIIIA1 in different levels

The above results indicate that mTOR plays a vital role in the expression of $A 1$ in MG-63 cells which received mechanical stress. Other studies have shown that ITORC1 phosphorylates S6K1 and 4E-BP1 to stimulate protein synthesis. Here, we further explore the role of S6K1 and 4E-BP1 on the expression of A1 mRNA or protein by qRT-PCR or Western blotting, as described above.

A1 mRNA showed the largest expression level when cells received mechanical stress for $12 \mathrm{~h}$, which was chosen for succedent research (Figs. 3 and 4). MG-63 cells were divided into 4 groups: control group (cells without any treatment) and three experimental groups. The latter includes a group which only received mechanical stress, a group pretreated with $4 \mathrm{E}-$ BP1 siRNA and mechanical stress, and a group pretreated with S6K1 siRNA and mechanical stress, respectively. At the mRNA level, the results showed that A1 mRNA was significantly upregulated in the group which only received mechanical stress. On the contrary, A1 mRNA was significantly down-regulated in the group that was pretreated with S6K1 siRNA and mechanical stress. Both were compared with the control group $\left({ }^{\star} \mathrm{P}<\right.$ $0.05)$. Also, A1 mRNA levels in the group pretreated with S6K1 siRNA and mechanical stress were significantly down-regulated compared with those in the group that only received mechanical stress ( $\left.{ }^{\#} \mathrm{P}<0.05\right)$ (Fig. 5A). At the protein level, the expression of $A 1$ was significantly up-regulated in the group that only

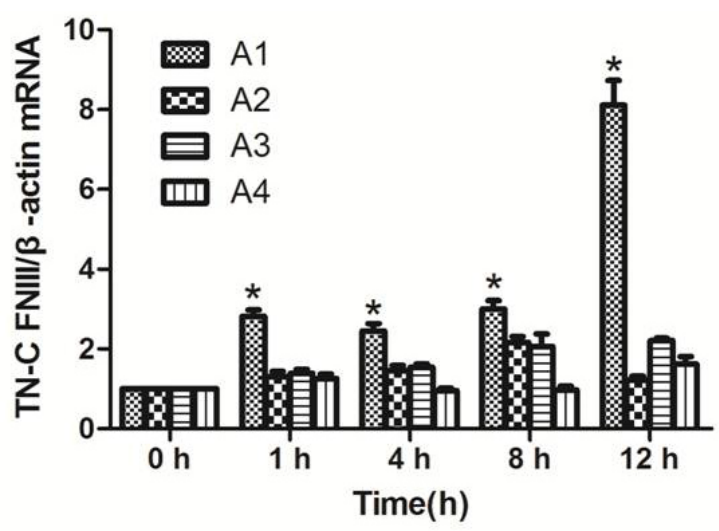

Fig. 3. The mRNA level of $A 1$ is elevated in MG-63 cells under mechanical stress. Expression of A1 in MG-63 cells was detected by qRT-PCR under different stimulation time for $1 \mathrm{~h}, 4 \mathrm{~h}, 8 \mathrm{~h}$, and $12 \mathrm{~h}$, respectively. Same experiments were carried out to detect $\mathrm{A} 2$, $\mathrm{A} 3$ and $\mathrm{A} 4$, which are the alternatively spliced FNIII repeats. Data were normalized based on the $\beta$-actin levels. ${ }^{*} P<0.05$ by Student's test when compared with control group.

received mechanical stress and down-regulated in the group pretreated with 4E-BP1 siRNA and mechanical stress as well as the group pretreated with S6K1 siRNA and mechanical stress compared with the control group (Fig. 5B). These results suggest that S6K1 is necessary during A1 mRNA normal expression in MG-63 cells undergoing mechanical stress. However, both 4E-BP1 and S6K1 are necessary during A1 normal expression at the protein level in MG-63 cells experiencing mechanical stress. 
A

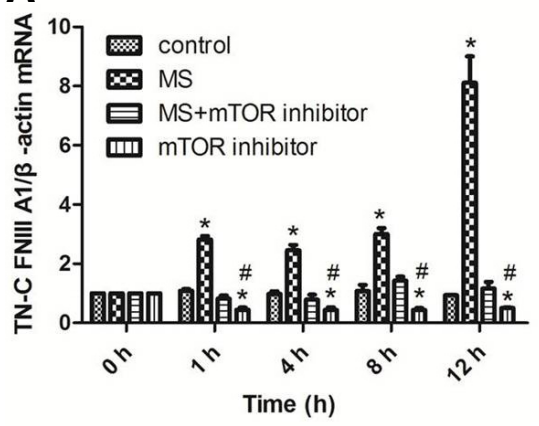

B
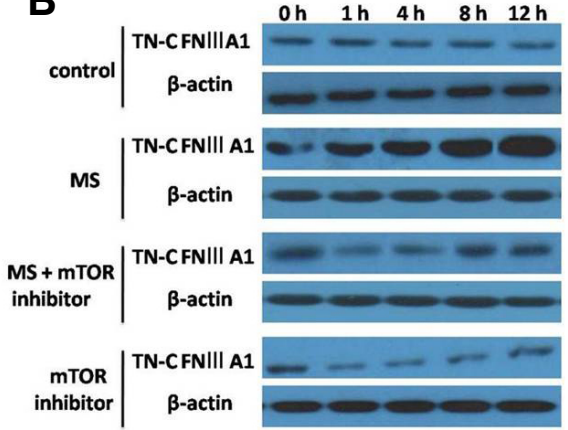

Fig. 4. Inhibition of mTOR decreases expression of TN-C FNIIIA1. A1 mRNA and protein expression in MG-63 cells treated by different conditions (mechanical stress, mTOR inhibitor or both) as measured by qRT-PCR and Western blotting. Data were normalized based on the $\beta$-actin levels. (A) The expression of A1 mRNA under different conditions by qRT-PCR. ${ }^{*} \mathrm{P}<$ 0.05 and ${ }^{\#} P<0.05$ by Student's test when compared with control group and group only received mechanical stress, respectively. (B) The protein level of $A 1$ under different conditions by Western blotting.
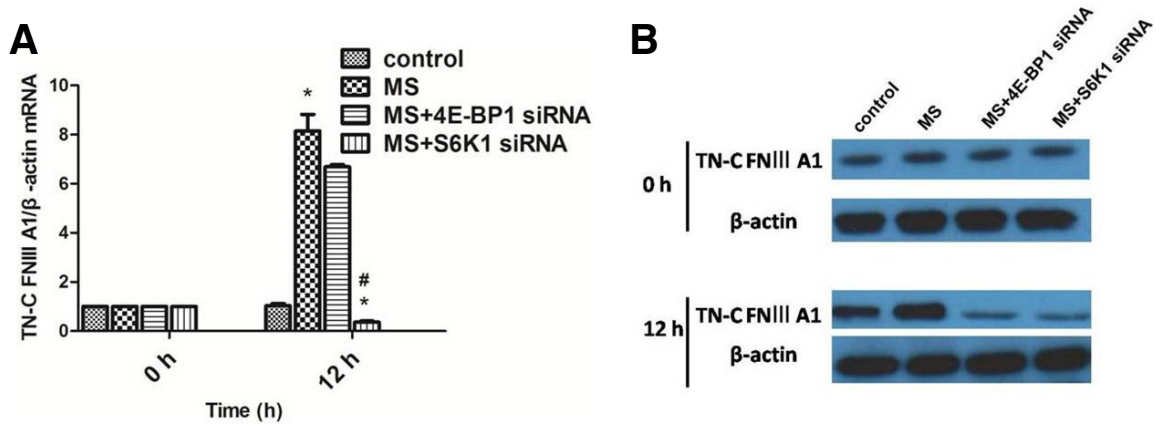

Fig. 5. Silencing of $4 \mathrm{E}-\mathrm{BP} 1$ and $\mathrm{S} 6 \mathrm{~K} 1$ decreases expression of TN-C FNIIIA1 in different levels. A1 mRNA and protein expression in MG-63 cells treated by different conditions (treated with mechanical stress only, treated with 4E-BP1 siRNA and mechanical stress, or treated with S6K1 siRNA and mechanical stress) were measured by qRT-PCR and Western blotting. Data were normalized based on the $\beta$-actin levels. (A) The expression

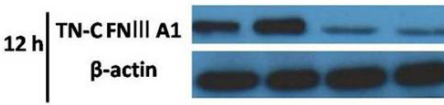

of $A 1$ mRNA in MG-63 cells treated by different conditions. ${ }^{*} P<0.05$ and ${ }^{\#} P<0.05$ by Student's test when compared with control group and group only received mechanical stress, respectively. (B) The expression of A1 in MG-63 cells treated by different conditions as detected by Western blotting.

\section{DISCUSSION}

Osteosarcoma (OS) accounts for $>10 \%$ of all solid cancers and $20-35 \%$ of all malignant primary bone tumors with a very poor prognosis (Bielack et al., 2009; Rhee et al., 2013). Surgery cannot achieve good clinical results because of its highly aggressive nature and the fact that it mostly metastasizes to the bones and lungs (Gill et al., 2012). Therefore, treating metastatic osteosarcoma remains a challenge for oncologists. Thus, a novel strategy to efficiently inhibit metastasis of osteosarcoma is highly indispensable.

Tenascin-C belongs to the family of extracellular matrix glycoproteins, which plays a vital role in cardiac and arterial injury, tumor angiogenesis and metastasis (Midwood et al., 2011; Sage and Bornstein, 1991; Tucker et al., 2006). Previous studies have reported the association between TN-C and tumors, and $\mathrm{TN}-\mathrm{C}$ is known to be overexpressed in various types of tumor cells, such as brain tumors (Midwood et al., 2011), oral squamous cell carcinoma (Ramos et al., 1998), and breast cancer (Jahkola et al., 1998).

Studies have demonstrated that mechanical stimulation can facilitate TN-C expression (Tucker and Chiquet-Ehrismann, 2009). In addition, TN-C can facilitate cell migration, and the alternatively spliced FNIII repeats of TN-C were shown to responsible for this effect (Midwood et al., 2011; Murphy-Ullrich, 2001; Tamaoki et al., 2005). Meanwhile, domain A1 of FNIII has become a research hotspot for the effect of inhibiting lymphocyte activation, tumor infiltrating lymphocytes, proliferation and blocking the secretion of cytokines (Midwood and Orend, 2009) However, it was still unknown whether mechanical stimulation can promote MG-63 cell migration though facilitating the overexpression of TN-C FNIIIA1. To the best of our knowledge, this is the first report to evaluate the usefulness of $A 1$ in the migration of MG-63 cells and its potential role in OS.

Through immunohistochemical analysis, we found that the A1 protein was present at a higher level in tissues of osteosarcoma patients than in adjacent normal tissues in the present study. Therefore, we constructed A1 overexpression and knockdown MG-63 cells, and the effect of this was verified by qRTPCR and Western blotting. Through cell migration assay, we observed that the number of MG-63 cells migrating into the denuded area increased in the order of $A 1$ knockdown MG-63 cells, MG-63 cells at rest and A1 overexpression MG-63 cells. These results suggested that TN-C FNIIIA1 can induce MG-63 cell migration in vitro and thus may play a critical role in OS metastasis. In addition, annexin II is a indispensable molecule during A1-overexpression mediated MG-63 cells migration, which may be the receptor for the alternatively spliced segment of TN-C FNIIIA1. In recent years, molecular target therapy has brought about a breakthrough in the treatment of tumors. Results presented in this study demonstrate that domain A1 of TN-C may be a potential therapeutic target in metastatic osteosarcoma. However, the clinical usefulness of $A 1$ in metastatic OS has not been fully described and further studies are needed to define the role of it in OS.

In order to explore the effect of mechanical stress on A1 ex- 
pression, we carried out mechanical stress experiments. According to our statistical analyses, mRNA levels of A1 compared with other domain of FNIII $(A 2, A 3, A 4)$ were significantly up-regulated with mechanical action time, especially in the $12 \mathrm{~h}$ group. This result suggests that mechanical stress derived from the daily activities of OS patients might promote the expression of A1. However, the detailed mechanism and its clinical reliability need to be confirmed. Therefore, we further explored the signaling pathway related to $A 1$ expression and found that the mTOR molecule is indispensable during this procedure. Meanwhile, further research about the effects of 4E-BP1 and S6K1 on $A 1$ expression in MG-63 cells experiencing mechanical stress demonstrated that 4E-BP1 and S6K1, which are downstream molecules of mTOR, are necessary for the normal expression of $A 1$ in MG-63 cells. Therefore, we speculated that mTOR, 4E-BP1 and S6K1 may be involved in the expression of $A 1$. This is consistent with the theory that mTORC1 phosphorylates S6K1 and 4E-BP1 to stimulate protein synthesis (Ma and Blenis, 2009; Zhang and Zheng, 2012).

For a long time, there has been no exercise limitation for OS patients clinically, due to the traditional view that there is no effect of exercise on OS. However, in our research, we demonstrated that mechanical stimulation can promote MG-63 cell migration though facilitating TN-C FNIIIA1 overexpression in vitro. This suggests that the daily activities of OS patients may promote the expression of $A 1$, and that the overexpression of A1 in OS tissues may influence OS migration and aggravate OS deterioration. Meanwhile, the results of this study provide the theoretical basis for the effect of $\mathrm{A} 1$ on OS migration in further clinical research. However, the clinical inhibition of activities in OS patients need to be further studied.

In conclusion, the high expression of $\mathrm{A} 1$ was correlated with MG-63 cell migration, suggesting that $A 1$ could be used as an indicator in metastatic osteosarcoma patients. Our results might provide a novel way to explore targeted therapy in OS patients. Furthermore, the effect of activities of OS patients on OS metastasis need further research.

\section{ACKNOWLEDGMENTS}

This work was supported by National Natural Science Funds for young scientists 810-0119-6 from central finance.

\section{REFERENCES}

Alves, T.R., da Fonseca, A.C.C., Nunes, S.S., da Silva, A.O., Dubois, L.G.F., Faria, J., Kahn, S.A., Viana, N.B., Marcondes, J., Legrand, C., et al. (2011). Tenascin-C in the extracellular matrix promotes the selection of highly proliferative and tubulogenesisdefective endothelial cells. Exp. Cell Res. 317, 2073-2085.

Andrianarivo, A.G., Robinson, J.A., Mann, K.G., and Tracy, R.P. (1992). Growth on type I collagen promotes expression of the osteoblastic phenotype in human osteosarcoma MG-63 cells. J. Cell. Physiol. 153, 256-265.

Bielack, S., Carrle, D., Casali, P.G., and Group, O.b.o.t.E.G.W. (2009). Osteosarcoma: ESMO Clinical Recommendations for diagnosis, treatment and follow-up. Ann. Oncol. 20, iv137-iv139.

Chung, C.Y., and Erickson, H. (1994). Cell surface annexin II is a high affinity receptor for the alternatively spliced segment of tenascin-C. J. Cell Biol. 126, 539-548.

Ehrlich, P., and Lanyon, L. (2002). Mechanical strain and bone cell function: a review. Osteoporosis Int. 13, 688-700

Feng, X., Zhang, Y., Xu, R., Xie, X., Tao, L., Gao, H., Gao, Y., He, Z., and Wang, H. (2010). Lipopolysaccharide up-regulates the expression of $\mathrm{Fc} \alpha / \mu$ receptor and promotes the binding of oxidized low-density lipoprotein and its IgM antibody complex to activated human macrophages. Atherosclerosis 208, 396-405.

Gill, J., Ahluwalia, M.K., Geller, D., and Gorlick, R. (2012). New targets and approaches in osteosarcoma. Pharmacol. Ther. 137, 89-99.
Hirata, E., Arakawa, Y., Shirahata, M., Yamaguchi, M., Kishi, Y., Okada, T., Takahashi, J.A., Matsuda, M., and Hashimoto, N. (2009). Endogenous tenascin-C enhances glioblastoma invasion with reactive change of surrounding brain tissue. Cancer Sci. 100, 1451-1459.

Jahkola, T., Toivonen, T., Virtanen, I., von Smitten, K., Nordling, S., von Boguslawski, K., Haglund, C., Nevanlinna, H., and Blomqvist, C. (1998). Tenascin-C expression in invasion border of early breast cancer: a predictor of local and distant recurrence. Br. J. Cancer 78, 1507.

Jiang, L., Wei, X., Yi, D., Xu, P., Liu, H., Chang, Q., Yang, S., Li, Z., Gao, H., and Hao, G. (2008). Synergistic effects of cyclic strain and Th1-like cytokines on tenascin-C production by rheumatic aortic valve interstitial cells. Clin. Exp. Immunol. 155, 216-223.

Leins, A., Riva, P., Lindstedt, R., Davidoff, M.S., Mehraein, P., and Weis, S. (2003). Expression of tenascin- $C$ in various human brain tumors and its relevance for survival in patients with astrocytoma. Cancer 98, 2430-2439.

Lelievre, S., Bisseil, M.J., and Pujuguet, P. (2000). Cell nucleus in context. Crit. Rev. Eukaryot. Gene Exp. 10, 30-37.

Li, R., Maminishkis, A., Wang, F.E., and Miller, S.S. (2007). PDGF$\mathrm{C}$ and-D induced proliferation/migration of human RPE is abolished by inflammatory cytokines. Invest. Ophthalmol. Vis. Sci. 48, 5722-5732.

Lv, Z., Yang, D., Li, J., Hu, M., Luo, M., Zhan, X., Song, P., Liu, C., Bai, H., and Li, B. (2013). Bone morphogenetic protein 9 overexpression reduces osteosarcoma cell migration and invasion. Mol. Cells 36, 119-126.

Ma, X.M., and Blenis, J. (2009). Molecular mechanisms of mTORmediated translational control. Nat. Rev. Mol. Cell Biol. 10, 307-318.

Midwood, K.S., and Orend, G. (2009). The role of tenascin-C in tissue injury and tumorigenesis. J. Cell Commun. Signal. 3, 287310.

Midwood, K.S., Hussenet, T., Langlois, B., and Orend, G. (2011) Advances in tenascin-C biology. Cell. Mol. Life Sci. 68, 3175-3199.

Murphy-Ullrich, J.E. (2001). The de-adhesive activity of matricellular proteins: is intermediate cell adhesion an adaptive state? J. Clin. Invest. 107, 785-790.

Oh, W.J., and Jacinto, E. (2011). mTOR complex 2 signaling and functions. Cell Cycle 10, 2305-2316.

Osborne, T., and Khanna, C. (2012). A review of the association between osteosarcoma metastasis and protein translation. $\mathrm{J}$. Comp. Pathol. 146, 132-142.

Pazzaglia, L., Conti, A., Chiechi, A., Novello, C., Magagnoli, G., Astolfi, A., Pession, A., Krenacs, T., Alberghini, M., and Picci, P. (2010). Differential gene expression in classic giant cell tumours of bone: tenascin $\mathrm{C}$ as biological risk factor for local relapses and metastases. Histopathology 57, 59-72.

Ramos, D.M., Chen, B., Regezi, J., Zardi, L., and Pytela, R. (1998). Tenascin-C matrix assembly in oral squamous cell carcinoma. Int. J. Cancer 75, 680-687.

Rhee, S.H., Han, I., Lee, M.R., Cho, H.S., Oh, J.H., and Kim, H.S. (2013). Role of integrin-linked kinase in osteosarcoma progression. J. Orthopaedic. Res. 31, 1668-1675.

Sage, E., and Bornstein, P. (1991). Extracellular proteins that modulate cell-matrix interactions. SPARC, tenascin, and thrombospondin. J. Biol. Chem. 266, 14831-14834.

Saito, Y., Imazeki, H., Miura, S., Yoshimura, T., Okutsu, H., Harada, Y., Ohwaki, T., Nagao, O., Kamiya, S., and Hayashi, R. (2007) A peptide derived from tenascin-C induces $\beta 1$ integrin activation through syndecan-4. J. Biol. Chem. 282, 34929-34937.

Shimoyama, T., Hiraoka, S., Takemoto, M., Koshizaka, M., Tokuyama, H., Tokuyama, T., Watanabe, A., Fujimoto, M., Kawamura $H_{\text {., }}$ and Sato, S. (2010). CCN3 inhibits neointimal hyperplasia through modulation of smooth muscle cell growth and migration. Arterioscler. Thromb. Vasc. Biol. 30, 675-682.

Si, Y., Wang, J., Guan, J., Han, Q., and Hui, Y. (2013). Plateletderived growth factor induced alpha-smooth muscle actin expression by human retinal pigment epithelium cell. J. Ocul. Pharmacol. Ther. 29, 310-318.

Skelly, D.T., Hennessy, E., Dansereau, M.-A., and Cunningham, C. (2013). A systematic analysis of the peripheral and CNS effects of systemic LPS, IL-1B, TNF- $\alpha$ and IL-6 challenges in C57BL/6 mice. PLoS One 8, e69123.

Tamaoki, M., Imanaka-Yoshida, K., Yokoyama, K., Nishioka, T., Inada, H., Hiroe, M., Sakakura, T., and Yoshida, T. (2005). 
Tenascin- $\mathrm{C}$ regulates recruitment of myofibroblasts during tissue repair after myocardial injury. Am. J. Pathol. 167, 71-80.

Tucker, R., Drabikowski, K., Hess, J., Ferralli, J., Chiquet-Ehrismann, R., and Adams, J. (2006). Phylogenetic analysis of the tenascin gene family: evidence of origin early in the chordate lineage. BMC Evol. Biol. 6, 60.

Tucker, R.P., and Chiquet-Ehrismann, R. (2009). The regulation of tenascin expression by tissue microenvironments. Biochim. Biophys. Acta $1793,888-892$.

Wachtel, M., and Schäfer, B.W. (2010). Targets for cancer therapy in childhood sarcomas. Cancer Treat. Rev. 36, 318-327.

Wang, Y., Man, Y., Ding, Y., Ma, B., Qiu, X., Fan, Q., Zheng, L. (2006). Mechanical strain and growth factors regulate expression of tenascin-C by OS cells additively. Oncol. Res. 20, 509-516.
Wang, Y., Zheng, L., Ma, B., Zhou, Y., and Fan, Q. (2010). Generation and identification of monoclonal antibodies against FNII domain D of human tenascin-C. Hybridoma (Larchmt) 29, 13-16.

Wu, G., Wang, J., Zhou, Z., Li, T., and Tang, F. (2013). Combined staining for immunohistochemical markers in the diagnosis of papillary thyroid carcinoma: Improvement in the sensitivity or specificity? J. Int. Med. Res. 41, 975-983.

Wullschleger, S., Loewith, R., and Hall, M.N. (2006). TOR signaling in growth and metabolism. Cell 124, 471-484.

Yuan, T., and Cantley, L. (2008). PI3K pathway alterations in cancer: variations on a theme. Oncogene 27, 5497-5510.

Zhang, Y., and Zheng, X.S. (2012). mTOR-independent 4E-BP1 phosphorylation is associated with cancer resistance to mTOR kinase inhibitors. Cell Cycle 11, 594-603. 